\title{
PURE POWERS ARE NOT POWERFUL QUALITIES
}

\author{
Joaquim Giannotti ${ }^{1}$ \\ ${ }^{1}$ University of Birmingham \\ Original scientific article - Received: 11/11/2020 Accepted: 27/01/2021
}

\begin{abstract}
There is no consensus on the most adequate conception of the fundamental properties of our world. The pure powers view and the identity theory of powerful qualities claim to be promising alternatives to categoricalism, the view that all fundamental properties essentially contribute to the qualitative make-up of things that have them. The pure powers view holds that fundamental properties essentially empower things that have them with a distinctive causal profile. On the identity theory, fundamental properties are dispositional as well as qualitative, or powerful qualities. Despite the manifest difference, Taylor (2018) argues that pure powers and powerful qualities collapse into the same ontology. If this collapse objection were sound, the debate between the pure powers view and the identity theory of powerful qualities would be illusory: these views could claim the same advantages and would suffer the same problems. Here I defend an ontologically robust distinction between pure powers and powerful qualities. To accomplish this aim, I show that the collapse between pure powers and powerful qualities can be resisted. I conclude by drawing some positive implications of this result.
\end{abstract}

Keywords: Pure powers; powerful qualities; dispositionalism; collapse objection; dispositional essentialism 


\section{The Qualitative and the Dispositional}

Fundamental properties are an elite minority that suffices to characterise all things completely and form a minimal basis on which all nonfundamental properties supervene (Lewis 1983, 1986, 2009). It is typically claimed that physics is in the business of discovering the fundamental properties of our world. Properties such as charge, mass, and spin are often invoked as plausible candidates. Yet there is no consensus on the most adequate conceptions of the fundamental properties of our world. The disagreement runs deep and covers several longstanding metaphysical questions (cf. Armstrong 2005). Here my focus is on whether the nature of fundamental properties is qualitative/categorical or dispositional.

Three monist views offer an answer to this question. Categoricalism holds that all fundamental properties are essentially and purely qualitative, or pure qualities (e.g., Lewis 1986; Armstrong 1997). The pure powers view holds that all fundamental properties are essentially and purely dispositional, or pure powers (e.g., Mumford 2004; Bird 2007a). The identity theory of powerful qualities holds that all fundamental properties have a dual nature: they are essentially both dispositional and qualitative, or powerful qualities (e.g. Martin 1993, 2008; Heil 2003, 2012). This view is committed to a distinctive three-fold identity claim: a fundamental property's dispositionality is identical with its qualitativity, and each of these is identical with the property itself (e.g., Heil 2003, 111; Taylor 2018, 1424). ${ }^{1}$

To elucidate these positions, we need two clarifications: one concerns what it is for a property to be a certain way, the other regards the notions of dispositionality and qualitativity.

For the sake of the discussion, let us assume that to say that a property $\mathrm{P}$ is essentially such-and-such means that it is true in virtue of $\mathrm{P}$ 's nature that $\mathrm{P}$ is such-and-such, or that P's nature grounds that P is such-and-such. As is now standard, if $\mathrm{P}$ is essentially such-and-such, then necessarily $\mathrm{P}$ is suchand-such, but the converse does not hold.

Now let us clarify dispositionality and qualitativity. Dispositionality is a matter of what a thing is disposed to do in various possible circumstances by virtue of having certain properties. Call these dispositional properties. We can think of dispositional properties as those that cannot be specified

\footnotetext{
${ }^{1}$ In the literature, we can find also mixed views. These hold that some fundamental properties are essentially qualitative, and others are essentially dispositional (e.g., Ellis 2001, 2002, 2012; Ellies and Lierse 1994). In what follows, I restrict my attention to monist views of fundamental properties.
} 
independently of any causal roles. These can be regarded as descriptions or ways of conceptualizing or specifications that typically refer to the manifestation of distinctive effects in distinctive circumstances. Accordingly, a property such as that of having a determinate charge is plausibly dispositional: it cannot be specified independently of the causal role of producing an electromagnetic force that electrons, say, play or possess. An essentially dispositional property, or power, is one for which it is true in virtue of its nature that it cannot be specified independently from any causal roles.

Some philosophers take qualitativity to be a matter of how a thing is in virtue of possessing some actual or occurrent properties (e.g., Strawson 2008, 278; Heil 2010, 70; Heil 2012, 59). However, on this understanding, every actual dispositional property would be qualitative. Such a result is unsavoury for those who wish to preserve the mutual exclusivity of dispositional and qualitative properties (e.g., Armstrong 2005; Bird 2007). Therefore, we need to opt for a different characterisation. Since my target in this paper is the powerful qualities view, the qualitative should be characterised in a way which permits one to coherently hold that a property is dispositional as well as qualitative. Consequently, I will not follow those who take the qualitative to be the non-dispositional. For example, I will part ways with Alexander Bird, who claims that a qualitative property requires us "to deny that it is necessarily dispositional" (2007a, 66-67).

Typically, examples of qualitative properties include shape and colour properties (being spherical, being scarlet), structural properties (having a determinate crystalline structure), geometrical properties (having an angle of a determinate measure), and spatio-temporal properties (having a determinate location). Two things group these properties: one is that they contribute to the make-up of objects that instantiate them, the other is that they can be specified independently of any causal roles. An instance of the property of having a tetrahedral molecular structure is plausibly qualitative for it contributes to the make-up of a bearer, say a diamond, and its characterisation does not force us to invoke any causal role. However, dispositional properties also contribute to the make-up of their bearers. For instance, the property of having a determinate charge is a part of the makeup of an electron. To draw the distinction, we should privilege the fact that qualitative properties can be specified independently from any causal roles. Accordingly, I submit that an essentially qualitative property, or quality, is one for which it is true in virtue of its nature that it can be specified independently from any causal role. Some ambiguity is nonetheless inescapable. For this reason, examples of qualities are contentious. I will mention properties such as that of having a certain quantity of charge and having a certain quantity of matter as plausible candidates of fundamental 
qualities (e.g., Giannotti 2019). These two quantitative properties seem to be exhaustively specified without any reference to causal roles and are more plausible fundamental properties than colours or geometrical shapes.

On this characterisation of the qualitative-dispositional distinction, some properties can be both dispositional and qualitative in the sense that they can be characterised (overtly or covertly) in terms of the causal as well as non-causal roles they play. It is one of the aims of this paper to clarify this view.

Two remarks on this characterisation of the qualitative-dispositional distinction are needed. First, it is not meant to be a reductive analysis of dispositionality and qualitativity. For the purposes of this paper, a general sense of these notions will suffice.

Second, this characterisation is not the only game in town. For example, it is orthodoxy amongst categoricalists and dispositionalists to define powers and qualities in mutually exclusive terms. But one is not forced to do so. As I explained, here we need to adopt a different conception of the qualitative and the dispositional. $^{2}$

\section{The Collapse Objection}

This paper concerns the pure powers view and the identity theory of powerful qualities. These views are manifestly distinct. It is one thing to claim that the nature of all fundamental properties is purely dispositional, however, it is another to claim that it is dispositional as well as qualitative. It seems that only the identity theory is prima facie committed to the view that fundamental properties are essentially dispositional as well as qualitative.

Contrary to the appearances, it has been recently argued that there is no real, ontologically robust distinction between the pure powers view and the identity theory of powerful qualities. Henry Taylor (2018) argues that they collapse into the same view. Call this the collapse objection.

If the collapse objection were sound, then the pure powers view and the identity theory could claim the same advantages and would suffer the same problems. The debate between these views would be illusory. Since each position claims to be preferable over other options in the debate about

\footnotetext{
${ }^{2}$ See Ingthorsson (2013) for an overview of various ways in which the qualitative-dispositional distinction is spelled out in the literature.
} 
fundamental properties, it is crucial to assess Taylor's collapse objection. My aim in this paper is to show that the collapse does not obtain: pure powers and powerful qualities share some relevant features and yet do not coincide. To do so, I defend an ontological demarcation between pure powers view and the identity theory.

Here is the plan. In the remainder of this section, I lay out a few assumptions that are needed for delineating the scope of this paper. In Section 3, I articulate the notion of a part of a property, which Taylor (2018) invokes to characterise the pure powers view and the identity theory. As it will become clear in due course, a suitable interpretation of this notion serves the purposes of this paper well. In Sections 4 and 5, I illustrate the pure powers view and the identity theory, respectively. In Section 6, I formulate the collapse objection in a more precise way. In Section 7, I show how to resist it by expanding on a strategy that I hinted at elsewhere (Giannotti 2019). I conclude in Section 8 by identifying some theoretical advantages of this result.

To begin with, let us acknowledge that Taylor's objection is not meant to undermine the prospects of dispositionalism tout court. Rather it is meant to show that two prominent dispositionalist approaches - namely, the pure powers view and the identity theory-fail to be ontologically distinct. Taylor offers his compound view, which I will outline in Section 4, as a positive alternative that preserves the dispositionalist spirit while escaping the collapse objection. If the argument in Section 7 is correct, we are not forced to embrace the compound view. This is good news for both the pure powers theorist and the identity theorist.

Second, it is not my aim to defend the correctness of either the pure powers view or the identity theory. It is one thing to show that the collapse between these views can be escaped. It is another thing to show that either of them is true. Here my focus is on the former task.

Third, the pure powers view and the identity theory can come in a variety of flavours. There are various ways of understanding the claim that powers are pure (e.g., Taylor 2018). Likewise, there are several ways of interpreting the claim that a property's dispositionality and its qualitativity are identical (I discuss some of these in Giannotti 2019). Furthermore, it is possible to articulate accounts that renounce the identity claim and yet share some similarities with powerful qualities (e.g., Tugby 2012; Taylor 2018; Giannotti 2019; Williams 2019). The discussion of the collapse objection is restricted to the versions of pure powers and powerful qualities that I present in what follows. However, I shall neglect the question of whether these versions are the best ones on the market. 
Bearing these remarks in mind, I will turn to illustrate Taylor's notion of a part of a property.

\section{3. "Parts" of Properties}

Taylor (2018) invokes the notion of a 'part' of a property to illustrate some of the claims that are attributed to both the pure powers view and the identity theory. For example, in describing the pure powers view, he says that "there is no part of a property's nature that is non-powerful" (2018, 1433). Unfortunately, Taylor does not elucidate what it is for a property to have parts. Let us assume that he makes no category mistakes. By doing so, we can articulate and evaluate a more fine-grained version of the collapse objection. We can also reformulate both the pure powers view and the identity theory in a way that illuminates the structure of fundamental properties as portrayed by these views. These advantages suggest that the notion of a part of a property is serviceable in casting a light on the "metaphysical workings" of pure powers and powerful qualities. However, my aim is not to offer a complete metaphysics of parts of properties. Some readers will find the choice of sticking with parts questionable and potentially confusing. The following remarks will address some initial reservations.

First, Taylor's notion of a part of a property is not mereological. The claim that a property has parts should not be understood as the claim that a property is made of more basic elements - parts - that constitute it. Therefore, talk of parts should not be construed as implying that fundamental properties are bundles or aggregates of parts. This mereological interpretation would threaten the claim that pure powers are fundamental ontological entities: arguably, if pure powers are composed of parts, these parts are more fundamental than the pure powers themselves.

There is another compelling reason for avoiding the mereological interpretation. If parts are themselves purely dispositional or purely qualitative properties, and if these are themselves made of parts, then a regress of parts emerges: the parts that make fundamental properties have further parts, which in turn have further parts, and so on ad infinitum. A somewhat similar problem occurs if we take parts as entailing the existence of other properties with parts: the latter would bring into existence further properties with parts, which in turn would bring into existence further properties with parts, and so on, ad infinitum again. These problematic consequences give us reasons for favouring a different approach. 
A more promising way of thinking of parts is to take them as features or aspects of fundamental properties that ground and explicate dispositional roles and qualitative features, where these roles and features do not entail the existence of other properties with parts. It is one of the aims of this paper to clarify this idea. Since the appeal to parts should preserve the view that pure powers are fundamental, parts are better regarded as being, in some sense, dependent upon the properties of which they are parts. Elsewhere, I proposed that parts can be thought of as aspects that are ontologically dependent upon properties (Giannotti 2019). In this paper, I wish to maintain a more flexible stance. We do not need to decide which relation better captures the link between parts and properties. Nor does the reader have to accept that parts of properties are the sort of aspects that I have in mind in Giannotti (2019).

In the literature, three views that adopt a similar conception of parts of properties are worthy of mention. In chronological order, the first one is the two-sided version of powerful qualities (Martin and Heil 1999). On this view, properties have dispositional and qualitative sides or aspects, which can be abstracted from the unitary property itself. For example, the property of having a determinate charge is two-sided in the sense that it can be thought of as a quality or power. We can regard it as the quality of having a specific quantity of charge or the disposition to produce an electromagnetic field. Another view is Tugby's (2012) qualitative dispositional essentialism. On this view, properties have a qualitative nature that grounds the dispositional aspect. The qualitative aspect is an inherent nature that grounds the causal roles associated with a property. Qualitative and dispositional aspects stand in a grounding relationship. Since it is the qualitative aspect of a property $\mathrm{P}$ that governs the causal roles that things play by virtue of instantiating P, Tugby's view is closer to the categoricalist camp than the dispositional one. The third view, as anticipated, is the dual-aspect account I put forward in Giannotti (2019). On this view, fundamental properties have dispositional and qualitative aspects that supervene on the property of which they are aspects and play distinct theoretical roles. ${ }^{3}$

The cited views are a few examples of how we can think of the relation between parts of properties-or aspects-and properties in nonmereological terms. They represent evidence of the plausibility of the idea

\footnotetext{
${ }^{3}$ Another philosopher who thinks that some properties have parts in a non-mereological sense is David Armstrong (1997). Structural universals have more basic universals as non-mereological constituents. Note, however, that if parts of properties are to be understood à la Armstrong, then these are presumably less fundamental then their parts. This conception is therefore inadequate for making sense of fundamental properties in terms of parts.
} 
that properties can have parts in a non-mereological sense, which is suitable to various ontological interpretations.

Equipped with parts of properties, let us move onto the discussion of pure powers

\section{Pure Powers}

The pure powers view holds that all fundamental properties are essentially purely dispositional, or pure powers (e.g., Mumford 2004; Bird 2007a, 2016). In this section, my aim is to illustrate Taylor's conception of pure powers, which I will adopt for the sake of the discussion.

At first glance, the purity claim seems to convey the idea that the dispositional nature of a pure power exhausts its being. As Taylor puts it, to say that a power is pure is to say that "the whole nature of a property is powerful: all of it is powerful and there is no part of a property's nature that is non-powerful" $(2018,1433)$. Crucially, this interpretation of the purity claim in terms of complete powerfulness is the one that Taylor endorses. He thinks of complete powerfulness as "the most natural way to interpret" the purity of pure powers $(2018,1433)$. By appealing to the notion of a part of a property, we can formulate the purity claim in terms of Complete Powerfulness, where Greek letters denote parts of properties:

Complete Powerfulness. For every part $\alpha$ of a fundamental property, $\alpha$ is dispositional.

This formulation captures the view that a pure power is completely powerful: since powers are essentially dispositional, it is in virtue of its nature that a pure power has only dispositional parts. Of course, we must supplement Complete Powerfulness with a characterisation of the notion of a dispositional part. Here is my preferred one.

\footnotetext{
Dispositional Part. A part $\alpha$ of a property P is dispositional if and only if there is a causal role or cluster of causal roles in virtue of $\alpha$ that is played or possessed by every object that has P.
}

The proposed characterisation regiments the idea that if a property has some dispositional parts, then an object instantiating it plays some causal roles by virtue of these parts. Differently put, the dispositional parts of a property are those that ground the causal roles of a bearer of that property. Tugby (2012) defends a similar characterisation: the dispositional aspects 
of a property are the causal roles that a bearer plays by virtue of instantiating that property. Here is an example to illustrate. Let us consider the property of having a determinate mass and the causal role of producing a gravitational force. Under Dispositional Part, if there is a part of the property of having a determinate mass such that any massive object plays the causal role of producing a gravitational force in virtue of it, then this part is dispositional. It is important to note that Dispositional Part is not meant to elucidate the notion of dispositionality. Instead, it expresses the relation between dispositional parts and causal roles. If we interpret the pure powers view in terms of Complete Powerfulness, this position holds that every part of the fundamental properties grounds the possession of some causal roles.

It is also important to stress that Taylor does not defend the claim that Complete Powerfulness is the only plausible interpretation of the purity claim. He makes a different claim, namely that Complete Powerfulness is the most natural interpretation of the claim that a power is purely dispositional. For the sake of the discussion, I will grant this point.

In addition to Complete Powerfulness, the version of pure powers view under scrutiny endorses two other claims. Let us call them Actuality and Non-Armstrongianism.

Actuality captures the idea that pure powers are actual, here-and-now properties of their bearers. As Taylor puts it, pure powers "are real, actual features of objects" $(2018,1431)$. We can formulate this claim as follows:

Actuality. Every fundamental property is an actual and real property of its bearers.

According to Actuality, if the property of having a determinate charge is a fundamental power, then it is also an actual and real property of its bearers.

Now let us consider Non-Armstrongianism. The pure powers theorist denies that fundamental properties are qualitative in the sense of being Armstrongian qualities, namely not essentially dispositional (e.g., Armstrong 1997). Here is one way to formulate this claim:

Non-Armstrongianism. Fundamental properties are not Armstrongian qualities.

Non-Armstrongianism allows us to distinguish the pure powers views (as thought of à la Taylor) from views like qualitative dispositional 
essentialism (Tugby 2012). On both views, certain parts of a property $\mathrm{P}$ ground the causal profile of something that instantiates P. Therefore, on both positions, the dispositional profile associated with $\mathrm{P}$ is necessary. However, the necessity flows from two incompatible sources. On the pure powers view, the dispositional profile is grounded in some dispositional parts; by contrast, on qualitative dispositional essentialism, it is grounded in some qualitative aspects.

To sum up, the version of the pure powers view that faces the collapse objection endorses three distinctive claims: Complete Powerfulness, Actuality, and Non-Armstrongianism. Now let us turn to illustrate the identity theory of powerful qualities.

\section{Powerful Qualities}

The identity theory holds that all fundamental properties have a dual nature: they are at once dispositional and qualitative, or powerful qualities (Martin 2008, 64). As Martin and Heil put it, "in virtue of possessing a property [powerful quality], an object possesses both a particular dispositionality and a particular qualitative character" $(1999,45-46){ }^{4}$

On this view, a fundamental property is essentially such that it can be characterised in terms of the causal roles played by things that instantiate it and the qualitative features these things have in virtue of it. With 'qualitative features', I have in mind features that can be described or conceptualized or specified without involving, overtly or covertly, any reference to manifestations of distinctive effects in characteristic circumstances.

Qualitative features are typically associated with qualities. Structural, geometrical, and mathematical features would be paradigmatic examples of qualitative features. If the property of having a determinate charge were a powerful quality, it would ground some causal roles, such as that of producing an electromagnetic field, and some qualitative features which something instantiating this property can be said to have. For example, it seems that by virtue of instantiating the property of having a determinate charge, objects can also be specified qualitatively in terms of having a certain quantity of charge (which can be measured in coulombs). To give another example, consider the property of having a determinate spin. If it were a powerful quality, it would ground some causal roles a bearer plays

\footnotetext{
${ }^{4}$ This formulation would make Tugby's (2012) qualitative dispositional essentialism a version of powerful qualities.
} 
and some of its qualitative features. For example, the causal role of producing a certain magnetic moment and the qualitative feature of having a specific quantity that can have only values that are multiples of $\hbar / 2$, where $\hbar$ is the reduced Planck constant.

We can appeal to Taylor's parts of properties to define the notion of a powerful quality in precise terms as follows.

Powerful Quality. A property $\mathrm{P}$ is a powerful quality if and only if $\mathrm{P}$ essentially has some dispositional parts and some qualitative parts.

The notion of a dispositional part is the same that has been introduced in Section 2. Now we need to characterise that of a qualitative part. A promising formulation, which captures the idea of qualitativity, is the following one.

Qualitative Part. A part $\alpha$ of a property P is qualitative if and only if there is a qualitative feature in virtue of $\alpha$ that is possessed by every object that has $\mathrm{P}$.

Put differently, a qualitative part of $\mathrm{P}$ is one which grounds the existence of a qualitative feature of a bearer of $\mathrm{P}$ which is neither overtly nor covertly dispositional. Suppose once again that the property of having a determinate charge is a powerful quality. If there is a part of this property in virtue of which a bearer has a feature that does not involve, either overtly or covertly, any manifestations of distinctive effects in characteristic circumstances, then this part is qualitative. To use the previous example, a qualitative part of the property of having a determinate charge is that which grounds a mathematical, non-dispositional feature of an electron, such as the possession of a certain quantity of charge that can be measured in coulombs. The powerful qualities theorist would maintain that the property of having a determinate charge has some dispositional parts in addition to this qualitative part. These ground the causal roles that the electron plays by virtue of having a determinate charge, such as that of producing an electromagnetic force. ${ }^{5}$ Putting these pieces together, we get that it is true in virtue of its nature that a powerful quality has some parts that ground some causal roles and some others that ground some qualitative features.

\footnotetext{
${ }^{5}$ Some caution is needed. We should not take Qualitative Part to be a definition of a quality. Otherwise, this definition would be problematically circular. Rather Qualitative Part simply captures the relation between the qualitativity of a bearer and some parts of a powerful quality which is instantiated by such a bearer.
} 
Someone could worry about inferring a robust distinction between dispositional and qualitative parts from a distinction between dispositional roles and qualitative features. Surely, it is one thing to describe a charged object in quantitative or mathematical terms, but it is another thing to describe it in causal or dispositional terms. However, such a distinction does not guarantee that such descriptions pick out different parts of a property of having a determinate charge. Instead of succumbing to this objection, identity theorists embrace the possibility that the same property can be at once dispositional as well as qualitative. To put it in terms of parts, identity theorists champion the idea qualitative and dispositional parts are, in a sense that I shall explain below, identical.

The identity theory endorses a distinctive three-fold identity claim between a property's dispositionality, its qualitativity, and the property itself (e.g., Heil 2003, 2012; Martin 2008; Taylor 2013). Heil formulates it as follows:

If $\mathrm{P}$ is an intrinsic property of a concrete object, $\mathrm{P}$ is simultaneously dispositional and qualitative; P's dispositionality and qualitativity are not aspects or properties of P; P's dispositionality, $\mathrm{Pd}$, is P's qualitativity, $\mathrm{Pq}$, and each of these is $\mathrm{P}: \mathrm{Pd}=\mathrm{Pq}=\mathrm{P}$. (Heil 2003, 111)

Difficulties in understanding this identity claim obfuscate the merits of powerful qualities. Here I do not wish to defend its correctness (for a discussion about some plausible interpretations, see Giannotti 2019). The clause "P's dispositionality and qualitativity are not aspects or properties of $\mathrm{P}$ " is meant to rule out the idea that powerful qualities are conjunctive properties made of purely dispositional and purely qualitative properties. Recall that on the proposed characterisation, parts are non-mereological aspects of properties that are dispositional and qualitative in virtue of playing the theoretical roles of grounding causal and qualitative features, respectively. Therefore, we should not think of dispositional and qualitative parts as purely dispositional and purely qualitative properties. Nor are these parts such that they bring into existence further purely dispositional or qualitative properties.

Now let us reformulate the identity claim in terms of parts of properties as follows.

Identity. For every fundamental property $\mathrm{P}$, (1) P has at least one dispositional part and $\mathrm{P}$ has at least one qualitative part, (2) every dispositional part of $\mathrm{P}$ is numerically identical with a qualitative part of $\mathrm{P}$ and vice versa, and (3) no part of $\mathrm{P}$ is a proper part. 
It is plainly obvious that Identity is different from Heil's formulation. However, the difference is not metaphysically deep: Identity preserves the original three-fold claim.

Clause (1) reformulates the claim that the properties are both dispositional and qualitative in terms of parts of properties.

Clause (2) does the same for the identity claim between a property's qualitativity and its dispositionality. Under the adoption of Dispositional Part and Qualitative Part, clause (2) says that every part of a property that grounds some causal roles is a part that also grounds some qualitative features. Take a particle that instantiates the property of having $a$ determinate mass. Under the assumption that this is a fundamental property, (2) implies that the part of this property that grounds the particle's causal role of producing a gravitational field is identical with the part that grounds one of the qualitative features of the particle, such as that of having a certain quantity of matter measurable in kilograms.

Clause (3) is a reformulation of the identity claim between a property's dispositionality and qualitativity and the property itself. The proposed interpretation borrows the proper/improper distinction from mereology to recover the idea that the dispositionality and qualitativity of a property are identical to the property itself. No proper part is identical with the object of which it is a part, but improper parts always are. It might be useful to acknowledge that views that endorse something like (3) already appeared in the literature. For example, Locke (2012) and Smith (2016) discuss versions of 'austere quidditism' and 'moderately austere quidditism' that take fundamental properties to be identical with and individuated by their qualitative suchness, which is an aspect of fundamental property (these views, however, differ with respect to the thinness of the qualitative aspect of fundamental properties). Smith (2016, 251-253) compares moderately austere quidditism with the identity theory explicitly. Smith's moderately austere quidditism holds that:

$[\ldots]$ the property and its qualitative nature are identical $(\mathrm{P}=$ $\mathrm{P}_{\mathrm{Q}}$ ), but the property and its dispositionality are plausibly distinct $\left(P \neq P_{D}\right)$ despite the fact that, as a matter of metaphysical necessity, an object instantiates $\mathrm{P}$ (and hence $\mathrm{P}_{\mathrm{Q}}$ ) if an only if it instantiates $P_{D}$. (Smith 2016, 252)

By contrast, as Identity states, the identity theory holds that a property and its dispositional parts are identical (that is, $\mathrm{P}=\mathrm{P}_{\mathrm{D}}$ ). I will return to Smith's view in the final section. 
Having outlined the pure powers view and the identity theory, we can now discuss the collapse objection.

\section{The Collapse Argument}

As Taylor notes, the identity theorist must embrace Complete Powerfulness $(2018,1434)$. Otherwise, a powerful quality would have some non-dispositional parts which would falsify Identity. Thus both the pure powers theorist and the identity theorist take fundamental properties to be completely powerful.

Like the pure powers theorist, the identity theorist also takes powerful qualities to be actual properties of their bearers. For example, Heil regards powerful qualities as qualities because they are "here and now, actual, not merely potential, features of objects, of which they are qualities" (2012, 59). This claim expresses a commitment to Actuality.

Lastly, on the identity theory, we must deny that fundamental powerful qualities are qualitative in the Armstrongian sense-namely, essentially non-dispositional. Otherwise, Identity could not hold. Thus the identity theorist embraces Non-Armstrongianism.

As I explained in Section 3, the pure power theorist holds Complete Powerfulness, Actuality, and Non-Armstrongianism. The pieces of the collapse objection are now put together. As Taylor puts it:

[...] The two views share the same commitments concerning the ontology of properties: both accept that properties are powers, both accept that they are 'qualities' in the same ways, and both accept the same interpretation of the 'purity' claims. (Taylor 2018, 1435)

In the remainder of the paper, I will show how to resist the collapse. I will explain that even if the pure powers view and the identity theory endorse Complete Powerfulness, Actuality, and Non-Armstrongianism, these views do not coincide because pure powers and powerful qualities are essentially distinct: not everything that is true in virtue of the nature of a pure power is also true in virtue of the nature of a powerful quality.

Before proceeding any further, it is worth stressing that the collapse objection targets only views of pure powers and powerful qualities, which endorse Complete Powerfulness, Actuality, and Non-Armstrongianism. A 
straightforward way to escape the collapse would be to adopt a view of fundamental properties which renounces one of these claims.

The rejection of Actuality seems to be the most problematic option though. It would imply that fundamental properties are not actual features of their bearers. Such a view should strike us as implausible.

The rejection of Non-Armstrongianism implies that fundamental properties are essentially non-dispositional qualities. If we follow this approach, both the pure powers view and the identity theory must be abandoned. Neither the pure powers theorist nor the identity theorist can contemplate this decision.

Something similar can be said for the option of giving up Complete Powerfulness. Because not all their parts would be dispositional, this solution would imply that fundamental properties are neither pure powers nor powerful qualities - at least as standardly construed. But nor would they automatically be Armstrongian qualities. The denial of Complete Powerfulness is, in fact, compatible with views that take fundamental properties to have both dispositional and qualitative, non-dispositional parts. ${ }^{6}$ These views, which I shall not discuss here, demand the acceptance of a new kind of properties. Surely, this will be a fair cost for some. However, before we pay it by the coin of ontology, it is worth exploring whether we can resist the collapse objection without abandoning Complete Powerfulness.

\section{Escaping the Collapse}

Elsewhere, I suggested that the identity theorist can argue that it is a "dual nature" (Martin and Heil 1999, 46; Martin 2008, 45; see also Giannotti 2019) that makes a powerful quality dispositional and qualitative; in contrast, a pure power has a powerful but not qualitative nature. Call this the distinct nature strategy.

Lamentably, Giannotti (2019) merely gestures toward the distinct nature strategy without offering a clear articulation. Since there I take the qualitative to be a matter of the actual contribution to the make-up of bearers, the lack of elucidation is problematic: it leads us to misleadingly think that pure powers are not qualitative in the sense of being actual (e.g., Taylor forthcoming). However, since I acknowledge that pure powers are

\footnotetext{
${ }^{6}$ For example, Taylor (2018, 1438-1439) offers a compound view of properties that have dispositional and qualitative, non-dispositional parts. Giannotti (2019) and Williams (2019) put forward similar views. For a critical discussion of Giannotti's dual-aspect account, see Taylor (forthcoming).
} 
actual, the manoeuvre is meant to take a different shape. In this section, I will endeavour to follow the distinct nature strategy through, thereby showing that it is indeed a promising option for resisting the collapse objection. The upshot of this strategy is that both the pure powers view and the identity theory endorse Complete Powerfulness, Actuality, and NonArmstrongianism and yet pure powers and powerful qualities have different natures - that is, they are essentially distinct.

The distinct nature strategy aims to establish the soundness of the following argument:

(1) If pure powers and powerful qualities are essentially distinct, then they are ontologically distinct kinds of properties.

(2) If pure powers and powerful qualities are ontologically distinct kinds of properties, then the pure powers view and the identity theory do not amount to the same view.

(3) Pure powers and powerful qualities are essentially distinct.

Therefore:

(4) The pure powers view and the identity theory do not amount to the same view.

If sound, this argument establishes that pure powers and powerful qualities do not "share the same commitments concerning the ontology of properties" (Taylor 2018, 1435). The conclusion (4) is the denial of the conclusion of the collapse argument, namely that pure powers and powerful qualities "are not distinct" (Taylor 2018, 1438). Importantly, (4) is compatible with the possibility that the pure powers view and the identity theory share some commitments. That is, (4) is consistent with Taylor's claim that the two views share Complete Powerfulness, Actuality, and Non-Armstrongianism.

The success of the distinct nature strategy hangs on premise (3). Recall that I adopted a conception of essentiality according to which the claim that a property $\mathrm{P}$ is essentially such-and-such means that it is true in virtue of $\mathrm{P}$ 's nature that $\mathrm{P}$ is such-and-such (Section 1). Accordingly, (3) states that pure powers and powerful qualities differ with respect to what is true in virtue of their own nature.

Both the pure powers view and the identity theory endorse Complete Powerfulness, which describes or characterises the nature of fundamental properties. Since both views endorse it, we should expect that Complete Powerfulness entails the same view about what it is essential to 
fundamental properties. If there is a difference between pure powers and powerful qualities in what is essential to them, then premise (3) is truenamely, it is true that pure powers and powerful qualities have different natures. The proposed framework of parts of properties is extremely serviceable for establishing this claim.

Let us start by observing that Complete Powerfulness entails the following characterisation of the nature of pure powers and powerful qualities:

Essential Dispositionality. For every essential part $\alpha$ of a fundamental property, $\alpha$ is dispositional.

This should be uncontroversial: if all parts of a power are dispositional, so are its essential parts. Essential Dispositionality says that every essential part of a fundamental property grounds some causal roles that bearers of such a property play. Both the pure powers theorist and the identity theorist happily accept Essential Dispositionality. According to the pure powers theorist, a power's nature is exhausted in its powerfulness (e.g., Bird 2007a, 100). The identity theorist also embraces Essential Dispositionality. A powerful quality does not comprise any non-dispositional parts; otherwise, Identity would be false (e.g., Heil 2003, 111). So far, so good. However, there is a crucial difference: only the identity theory is committed to Identity, namely the claim that the dispositional and qualitative parts of a property are identical. And the conjunction of Identity and Essential Dispositionality entails another claim:

Essential Qualitativity. For every essential part $\alpha$ of a fundamental property, $\alpha$ is qualitative.

This claim is true of powerful qualities. It states that every essential part of a fundamental powerful quality grounds some qualitative features that bearers of such a powerful quality has. Essential Dispositionality and Essential Qualitativity taken together capture the spirit of the identity theory nicely (e.g., Martin and Heil 1999, 45-46): by virtue of the essential parts of a fundamental powerful quality $\mathrm{P}$, every object that instantiates $\mathrm{P}$ has a particular dispositionality and a particular qualitativity. Note that the identity theorist cannot separate Essential Dispositionality and Essential Qualitativity. By embracing Identity, Complete Powerfulness gives the identity theorist a two-for-one deal: Identity and Complete Powerfulness entail both Essential Dispositionality and Essential Qualitativity.

The pure powers theorist does not embrace Identity. But it is only under the assumption of Identity that Complete Powerfulness entails both Essential Dispositionality and Essential Qualitativity. Therefore, on the 
pure powers view, Complete Powerfulness does not entail Essential Qualitativity. This seems quite right: the pure powers view denies that the essence of fundamental powers is to ground qualitative, non-causal features of bearers.

It appears, therefore, that Essential Dispositionality and Essential Qualitativity are both true of the identity theory. And Essential Dispositionality is true of the pure powers view. But Essential Qualitativity is not true of the pure powers view. Nor does it capture the nature of pure powers. Premise (3) of the distinct nature argument is consequently true: pure powers and powerful qualities are essentially distinct. The collapse is escaped. We can draw an ontological demarcation between the pure powers view and the identity theory on the grounds of their difference with respect to the truth of Essential Qualitativity.

Someone might worry that the distinct nature argument jeopardizes the robustness of the qualitative-dispositional distinction. However, the identity theorist would embrace this result. If the identity theory is true, then the difference between dispositional and qualitative parts does not demarcate a real distinction among properties. The identity theorist would stress that there is no incoherence in claiming that the same part of a property can ground some causal roles and some qualitative features. Yet the proposed characterisation of dispositional and qualitative parts does not automatically establish that this is indeed the case.

Here I do not wish to establish the correctness of the identity theory. So, I will just hint at a possible strategy to show that the qualitative and dispositional parts of a property are indeed identical. Distinctness of roles, the identity theorist could argue, does not reflect distinctness of parts. Just as the same person can play the role of a parent and of a Judge of the Supreme Court, so could the same part play both the role of grounding some causal roles and of grounding qualitative features. I leave the task of fleshing out this argument and the discussion of potential objections to a separate work.

For now, let us focus on the implications of the distinct nature strategy. If someone does not adopt Identity, then Essential Dispositionality and Essential Qualitativity do not entail each other. Therefore, someone could endorse one thesis while rejecting the other. For instance, the pure powers theorist can accept Essential Dispositionality while denying Essential Qualitativity. Presumably, the categoricalist embraces Essential Qualitativity while rejecting Essential Dispositionality (e.g., Lewis 1986; Armstrong 1997). For example, the categoricalist would say that by virtue of parts that belong to the property of having a determinate charge, 
an electron has some qualitative feature such as that of having a certain quantity of charge that can be measured in coulombs. In contrast, the electron's dispositionality would obtain in virtue of something distinct from the property itself. For example, it could hold in virtue of some laws of nature (e.g., Armstrong 1997).

The above considerations vindicate the claim that pure powers and powerful qualities are essentially distinct. Only the identity theory appears to be committed to both Essential Dispositionality and Essential Qualitativity. In contrast, the pure powers view appears to be committed only to Essential Dispositionality. Such a difference between the pure powers view and the identity theory strongly suggests that pure powers and powerful qualities have different natures: every part of a powerful quality grounds both causal roles and qualitative features; by contrast, every part of a pure power grounds causal roles only.

Before we move on, however, it is important to emphasise that the failure of the collapse between pure powers and powerful qualities does not imply that Taylor's (2018) considerations are wholly incorrect. He is right in thinking that the pure powers view and the identity theory share some commitments about the ontology of fundamental properties. The mistake, if I am right, is to infer the collapse between these views from these shared commitments.

Against the distinct nature strategy, someone might argue that the denial of Essential Qualitativity undermines the Actuality of pure powers (Taylor forthcoming raises a similar objection against Giannotti's dualaspect account). Therefore, this approach would render the pure powers view implausible.

Here is one way to spell out this objection: if the nature of a pure power does not contribute to the qualitativity of an object that instantiates it, then nothing secures the reality of such a pure power. For instance, an opponent could argue that if having a determinate charge were not to contribute to an electron's qualitative make-up, then nothing would ground the actuality and reality of this property. The threat would extend to every putative fundamental power.

An easy way out would be to embrace Essential Qualitativity. Accordingly, it would be part of the nature of fundamental pure powers to ground some qualitative features had by a bearer, thereby securing the reality of such powers. Unfortunately, this option opens the door to the collapse. If Essential Qualitativity were true of the pure powers view, 
then the distinct nature strategy would fail; the pure powers view would indeed coincide with the identity theory.

The previous objection barks but does not bite. Surely, the pure powers theorist must safeguard Actuality. However, it is a mistake to think that Essential Qualitativity is the only way to do so. Recall that Essential Qualitativity is a claim about the qualitative features that are grounded by some parts of a property. Of course, if one conceives of the qualitative features of an object as a matter of its actual make-up, then Essential Qualitativity ensures the actuality of the relevant properties. However, on the proposed characterisation, Actuality and Essential Qualitativity come apart. The former is a claim about the possession of powers; the latter concerns their nature. The possibility of holding Actuality without Essential Qualitativity is good news for the pure powers theorist who wishes to escape the collapse objection once and for all.

One promising option to ground Actuality, which escapes the collapse objection, is to argue that it is the possession of a power by a bearer that makes it actual and real. Differently put, what grounds the reality and actuality of a pure power is the fact that it is possessed by some object. This approach captures what George Molnar says by claiming that powers are actual and real in the sense that "having a power is ... having an actual property" (2003, 99). Thus Actuality should not be confused with a claim about the nature of pure powers.

Now I turn to conclude by pointing out some positive implications of the distinct nature strategy for both the pure powers view and the identity theory.

\section{Divide et Impera}

It goes without saying that by resisting the collapse objection, we make the dispute between the pure powers view and the identity theory substantive again. However, both positions also enjoy less obvious merits and drawbacks that concern their opposite stance on Essential Qualitativity. In what follows, I will point out an issue that the pure powers theorist can prima facie escape by rejecting Essential Qualitativity. To level the playing field, I will then consider an objection against pure powers that the identity theorist can prima facie avoid by embracing Essential Qualitativity. I do not aim to adjudicate which views handle these objections better. Nor is my aim to establish that these issues fatally wound either position. Rather, my purpose is to show that the ontological distinction between pure powers and powerful qualities has important consequences regarding the advantages of either doctrine. 
The pure powers view is often introduced as a form of anti-quidditism (e.g., Bird 2007, 70-79). Since there is no univocal understanding of what quidditism is, the opposition can be construed in a variety of ways. Here I shall not attempt to reconstruct the debate surrounding this notion. Others have already done so meticulously (e.g., Locke 2012, Smith 2016, Wang 2016). Instead, let us consider again moderately austere quidditism (Section 5)-according to which fundamental properties are individuated by a qualitative nature (Smith 2016, 250). This view allows for causally indistinguishable possible worlds that differ just by a permutation or replacement of fundamental properties. For example, there can be possible worlds where the causal roles played by our worldly charge and mass are the same and yet the properties that play such roles are swapped. ${ }^{7}$ Pure powers theorists argue that we should block possibilities of this sort.

The rejection of Essential Qualitativity secures this result. Fundamental pure powers lack qualitative parts. So, they also lack qualitatively quidditistic aspects which could individuate them. Pure powers are individuated by their essential causal roles which are, on the proposed framework, grounded in their dispositional parts. By contrast, the identity theory faces an odd consequence. Under the assumption that the quidditistic nature of a property is a matter of its qualitativity, powerful qualities would turn out to have quidditistic parts because of Essential Qualitativity. Perhaps shockingly, the identity theory would emerge as a form of quiddistim. In fairness, it is worth noting that the identity theory would block worrisome scenarios where causal roles are swapped (the identity between qualitative and dispositional parts would prevent the swapping). However, the worry that there is something odd about this upshot remains: typically, quidditistic features are supposed to be nondispositional (Smith 2016, 252). Perhaps the identity theorist could insist that the felt sense of oddity is a remnant of an ill-conceived attachment to the idea that dispositionality and qualitativity are mutually exclusive. Be that as it may, facing the odd consequence is a drawback that the pure powers view easily avoids.

The rejection of Essential Qualitativity is not without problems, however. For example, E. J. Lowe argues that an ontology of nothing but pure powers cannot fix the identity of fundamental properties (2010, 12-14; 2012, 217-228). Lowe's objection is as follows. What individuates, and therefore identifies, pure powers is their causal roles. But from the

\footnotetext{
${ }^{7}$ Not all form of moderately austere quidditism entail this possibility. For example, Smith's (2016) non-recombinatorial version imposes necessary connections between qualitative natures and their causal roles, thereby blocking swapped scenarios akin to the one illustrated here.
} 
viewpoint of an ontology of nothing but pure powers, the causal roles involve other pure powers. In turn, these will be individuated by some causal roles which involve further pure powers. And on it goes. The problem, Lowe claims, is that pure powers cannot get their identity fixed if they owe it to other ones. According to Lowe, the metaphysics of pure powers lack the resources for accommodating suitable individuators of properties, which ought to be qualitative features. Of course, Lowe's objection as well as his claim about qualitative individuators can be challenged. But suppose, for the sake of argument, that Lowe is right. Does the identity theory suffer the same problem?

It does not seem so. By embracing Essential Qualitativity, the identity theorist can locate Lowe's individuators in the qualitative features that bearers of fundamental powerful qualities have by virtue of instantiating them. These qualitative features do not involve the acquisition of pure powers. Therefore, the inadmissible regress of identity is blocked from the get-go. At least in principle, the identity theory does have the resources for meeting Lowe's challenge. Since the pure powers theorist cannot pursue the same strategy, the identity theorist can claim an advantage.

A lot more could be said about the previous objections and how to address them. But my goal is not to adjudicate a winner between pure powers and powerful qualities. The lesson here is that there are objections that target only one view but not the other. An explanation of this fact, if I am right, lies in the different commitments about the metaphysics of fundamental properties, particularly concerning Identity and Essential Qualitativity, that these views endorse. The discrepancy is beneficial. If one of the above objections were to be lethal for one view, the other could still be available. Overall, the possibility of retaining a robust ontological distinction between pure powers and powerful qualities is advantageous for the advocates of both theories.

\section{Acknowledgements}

Thanks to María Pía Méndez Mateluna, Rani Anjum, Lorenzo Azzano, Donatella Donati, Toby Friend, Giacomo Giannini, Stephen Mumford, Andrea Raimondi, Matthew Tugby, Barbara Vetter, Lisa Vogt, Neil E. Williams, a set of anonymous reviewers for helpful discussion on various parts of this manuscript, and to the audience of the New Foundations of Dispositionalism conference in Exeter. 


\section{REFERENCES}

Anjum, Rani Lill, and Stephen Mumford. 2011. Getting Causes from Powers. Oxford: Oxford University Press.

Armstrong, David M. (1978). Universals and Scientific Realism: A Theory of Universals Volume 2. Cambridge: Cambridge University Press.

Armstrong, David M. 1989. A Combinatorial Theory of Possibility. Cambridge: Cambridge University Press.

Armstrong, David M. 1997. A World of States of Affairs. Cambridge: Cambridge University Press.

Armstrong, David. M. 2005. 'Four Disputes about Properties'. Synthese 144: 309-320.

https://doi.org/10.1007/s11229-005-5852-7

Bird, Alexander. 2007a. Nature's Metaphysics. New York: Oxford University Press.

Bird, Alexander. 2007b. 'The Regress of Pure Powers?'. The Philosophical Quarterly 57(229): 513-534. https://doi.org/10.1111/j.1467-9213.2007.507.x

Bird, A. 2016. 'Overpowering: How the Powers Ontology Has Overreached Itself'. Mind 125(498): 341-383. https://doi.org/10.1093/mind/fzv207

Ellis, Brian. 2001. Scientific Essentialism. Cambridge: Cambridge University Press.

Ellis, Brian. 2002. The Philosophy of Nature. Chesham: Acumen.

Ellis, Brian. 2012. 'The Categorical Dimensions of the Causal Powers'. In Properties, Powers and Structures, edited by Alexander Bird, Brian Ellis and Howard Sankey, 11-27. New York: Routledge.

Ellis, Brian and Caroline Lierse. 1994. 'Dispositional Essentialism'. Australasian Journal of Philosophy 72(1): 27-45. https://doi.org/10.1080/00048409412345861

Giannotti, Joaquim. 2019. 'The Identity Theory of Powers Revised'. Erkenntnis: 1-19.

https://doi.org/10.1007/s10670-019-00122-5

Heil, John. 2003. From an Ontological Point of View. Oxford: Oxford University Press.

Heil, John. 2010. 'Powerful Qualities'. In The Metaphysics of Powers: Their Grounding and their Manifestations, edited by Anna Marmodoro, 58-73. New York: Routledge.

Heil, John. 2012. The Universe as We Find It. Oxford: Oxford University Press.

Lewis. David K. 1983. 'New Work for a Theory of Universals'. Australasian Journal of Philosophy 61(4): 343-377. https://doi.org/10.1080/00048408312341131 
Lewis, David K. 1986. On the Plurality of Worlds. Oxford: Blackwell Lewis, David K. 2009. 'Ramseyan humility'. In Conceptual Analysis and Philosophical Naturalism, edited by David Braddon-Mitchell and Robert Nola, 203-222. Cambridge: MIT Press. https://doi.org/10.7551/mitpress/9780262012560.003.0009

Locke, Dustin. 2012. 'Quidditism Without Quiddities'. Philosophical Studies 160: 345-363. https://doi.org/10.1007/s11098-0119722-5

Lowe, E. Jonathan. 2010. 'On the Individuation of Powers'. In A. In The Metaphysics of Powers: Their Grounding and their Manifestations, edited by Anna Marmodoro, 8-26. New York: Routledge. https://doi.org/10.4324/9780203851289

Lowe, E. Jonathan. 2012. 'Asymmetrical Dependence in Individuation'. In Metaphysical Grounding: Understanding the Structure of Reality, edited by Fabrice Correia and Benjamin Schnieder, 214234. Cambridge: Cambridge University Press.

https://doi.org/10.1093/analys/anu046

Ingthorsson, Rögnvaldur. 2013. Properties: Qualities, Powers, or Both? Dialéctica 67(1): 55-80. https://doi.org/10.1111/1746-8361.12011

Martin, Charles B. 1993. The Need of Ontology: Some Choices. Philosophy: The Journal of the Royal Institute of Philosophy 68(266): 502-522. https://doi.org/10.1017/s0031819100041863

Martin, Charles B. 2008. The Mind in Nature. Oxford: Oxford University Press.

Martin, Charles B., and Heil, John. 1999. The Ontological Turn. Midwest Studies in Philosophy 23(1): 34-60. https://doi.org/10.1111/1475-4975.00003

Molnar, George. 2003. Powers. New York: Oxford University Press.

Mumford, Stephen. 2004. Laws in Nature. London: Routledge.

Schaffer, Jonathan. 2005. 'Quiddistic Knowledge'. Philosophical Studies 123: $1-32$.

https://doi.org/10.1007/s11098-004-5221-2

Smith, Deborah. 2016. 'Quid Quidditism Est?'. Erkenntnis 81(2): 237257.

https://doi.org/10.1007/s10670-015-9737-y

Strawson, Galen. 2008. The Identity of the Categorical and the Dispositional. Analysis 68(4): 271-282.

https://doi.org/10.1093/analys/68.4.271

Taylor, Henry 2018. Powerful Qualities and Pure Powers. Philosophical Studies 175(6): 1423-1440.

https://doi.org/10.1007/s11098-017-0918-1 
Taylor, Henry. Forthcoming. Powerful Problems for Powerful Qualities. Erkenntnis. https://doi.org/10.1007/s10670-019-00199-y

Tugby, Matthew. 2012. Rescuing Dispositionalism from the Ultimate Problem: Reply to Barker and Smart. Analysis 72 (4): 723-731. https://doi.org/10.1093/analys/ans112

Yates, David 2012. 'The Essence of Dispositional Essentialism'. Philosophy and Phenomenological Research 87(1): 93-28. https://doi.org/10.1111/j.1933-1592.2011.00568.x

Wang, Jennifer 2016. 'The Nature of Properties: Causal Essentialism and Quidditism'. Philosophy Compass 11 (3): 168-176. https://doi.org/10.1111/phc3.12307

Williams, Neil E. 2019. The Powers Metaphysics. New York: Oxford University Press. 
